\title{
Extraction of Trivalent Rare Earth Ions with an Acidic Tridentate Extractant, 6- $N, N$-Di- $n$-Octylcarbamoyl-Pyridine-2-Carboxylic Acid
}

\author{
Hirokazu NARITA $^{1, *}$ and Mikiya TANAKA ${ }^{2}$ \\ ${ }^{1}$ Global Zero Emission Research Center, National Institute of Advanced Industrial Science and Technology \\ (AIST), 16-1 Onogawa, Tsukuba 305-8569, Japan; ${ }^{2}$ Tsukuba West, AIST Tsukuba, National Institute of \\ Advanced Industrial Science and Technology (AIST), 16-1 Onogawa, Tsukuba 305-8569, Japan
}

(Received February 3, 2021; Accepted February 22, 2021)

Extraction properties of trivalent rare earth ions (RE(III)) from acidic chloride solutions with 6- $N, N$-di- $n$-octylcarbamoyl-pyridine-2-carboxylic acid (CPCA), which has three functional groups: an amide, a carboxylic acid and a pyridine, were investigated by slope analyses of the distribution ratio $(D)$ of $\mathrm{RE}(\mathrm{III})$ and FT-IR measurements. The dependence of $D$ on $\mathrm{pH}$ and CPCA concentration indicated that the 1:3 RE(III)-CPCA complex was predominantly formed in the organic phase. FT-IR analysis of the Dy(III) complex extracted with CPCA suggested that the coordination mode of CPCA to Dy(III) was tridentate. Plots of extraction percentage ( $E \%)$ of $\mathrm{RE}(\mathrm{III})$ versus atomic number revealed that $E \%$ increased from $\mathrm{La}(\mathrm{III})$ to $\mathrm{Er}(\mathrm{III})$ and then remained almost constant until $\mathrm{Lu}(\mathrm{III})$. The $E \%$ of Y(III) was similar to that of $\mathrm{Sm}(\mathrm{III})$.

\section{Introduction}

The extraction of rare earth (RE) elements has recently attracted increased attention because of the industrial importance of RE elements and the vulnerability of their supply chain [1-4]. Commercially, extraction of RE elements is accomplished with extractants such as tri- $n$-butyl phosphate, quaternary ammonium compounds (e.g., Aliquat 336), tertiary carboxylic acids (e.g., Versatic acid), di-2-ethylhexyl phosphoric acid (D2EHPA), and 2-ethylhexylphosphonic acid mono-2-ethylhexyl ester (HEHEHP, also known as PC-88A) [5-7]. Among these extractants, D2EHPA and HEHEHP are effective for the mutual separation of the heavy lanthanides [1].

Various fundamental studies examining the extraction of RE(III) using amide-type extractants have been reported [8-15]. For example, tridentate diglycolamide compounds, which have two $N, N$-disubstituted amides and an ether group, show high extraction efficiency for $\mathrm{RE}(\mathrm{III})$ in $\mathrm{HNO}_{3}$ solutions, compared with other oxygen-donor bi- and tetra-dentate amide extractants $[9,10]$. A similar tridentate diamide, pyridine-2,6-dicaboxyamide, which has a pyridine group instead of an ether group, also shows high extraction efficiency for $\mathrm{RE}(\mathrm{III})$ in $\mathrm{HNO}_{3}$ solutions [11]. These diamide-type compounds cannot effectively extract $\mathrm{RE}(\mathrm{III})$ from dilute acidic solutions (e.g., $\mathrm{pH}>1$ ) because of neutral extractants. In contrast, a tridentate $N, N$-dioctyldiglycolamic acid (DGAA) can quantitatively extract RE(III) at $\mathrm{pH} 2-3$ and it shows a better mutual separation property for light lanthanides than both D2EHPA and Versatic acid 10 [12-14]; the combination of carboxyl, ether, and $N, N$-disubstituted amide groups appears to underlie its excellent 
extraction property. To understand the role of these donor atoms in detail, comparison with an acidic tridentate extractant having other donor atoms can be valuable.

Here, we examined the RE(III) extraction behavior using 6- $N, N$-di-n-octylcarbamoyl-pyridine-2-carboxylic acid (CPCA; Figure 1), which is a pyridine-containing acidic amide. Based on our findings, we discuss the extraction mechanism and the variation in extraction efficiency with increasing atomic number.

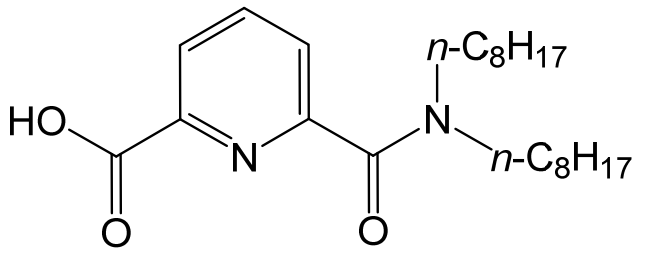

Figure 1. Structure of CPCA.

\section{Experimental}

\subsection{Reagents}

CPCA (purity: 99.8\%) was purchased from Wako Chemicals. Stock solutions of RE(III) were prepared by dissolving $\mathrm{RE}(\mathrm{III})$ chloride hydrates (Wako Chemicals) in dilute $\mathrm{HCl}$ solutions. All the chemicals used in this study were of reagent grade.

\subsection{Solvent extraction}

An aqueous mixed solution containing Nd(III), Eu(III), Dy(III), Lu(III) and Y(III) (5 × $10^{-4} \mathrm{M}$ each) was prepared by dissolving the relevant stock solutions in a $\mathrm{NaCl}$ solution; the ionic strength was adjusted to $1.0 \mathrm{M}(\mathrm{H}, \mathrm{Na}) \mathrm{Cl}$ using $\mathrm{HCl} / \mathrm{NaOH}$ solutions. Then the aqueous solution was added to the same volume of a chloroform solution containing CPCA, and the mixture was shaken vertically for $1 \mathrm{~h}$, which was enough time to reach the extraction equilibrium, and then centrifuged. After extraction, the $\mathrm{pH}$ and metal concentrations in the aqueous phase were measured by using a pH meter (Mettler Toledo, S220) and ICP-AES (Horiba, ULTIMA2), respectively, and the metal concentrations in the organic phase were calculated using the mass balance of the metal ions before and after the extraction. For the experiment on the effect of CPCA concentration (see Figure 2B), two kinds of aqueous solutions $(1.0 \mathrm{M}(\mathrm{H}, \mathrm{Na}) \mathrm{Cl}$ ) were used: a solution at $\mathrm{pH}>5$ containing $\mathrm{Nd}(\mathrm{III})$ and $\mathrm{Eu}(\mathrm{III})\left(5 \times 10^{-4} \mathrm{M}\right.$ each $)$ and a solution at $\mathrm{pH} \sim 2$ containing Dy(III), $\mathrm{Lu}(\mathrm{III})$, and $\mathrm{Y}(\mathrm{III})\left(5 \times 10^{-4} \mathrm{M}\right.$ each). The equilibrated $\mathrm{pH}$ after the metal extraction was $2.80(0.02), 2.67(0.03), 2.53(0.05), 2.39(0.10), 2.31(0.15), 2.27(0.20)$ for the former, and 2.15 (0.02), $2.13(0.03), 2.10(0.05), 2.02(0.10), 1.98(0.15)$ for the latter: the molar concentration of CPCA is given in parentheses. Extraction percentage $(E \%)$ and distribution ratio $(D)$ were calculated using the equations given below, where $[\mathrm{M}]_{\text {init,aq }},[\mathrm{M}]_{\mathrm{eq}, \mathrm{org}}$, and $[\mathrm{M}]_{\mathrm{eq}, \mathrm{aq}}$ denote the metal concentration in the initial aqueous phase, the equilibrated organic phase after extraction, and the equilibrated aqueous phase after extraction, respectively:

$$
\begin{aligned}
& \left.E \%=\left([\mathrm{M}]_{\text {eq,org }} /[\mathrm{M}]_{\text {init,aq }}\right) \times 100=\left\{\left([\mathrm{M}]_{\text {init,aq }}-[\mathrm{M}]_{\text {eq,aq }}\right) /[\mathrm{M}]_{\text {init,aq }}\right)\right\} \times 100 \\
& D=[\mathrm{M}]_{\text {eq,org }} /[\mathrm{M}]_{\text {eq,aq }}=\left([\mathrm{M}]_{\text {init,aq }}-[\mathrm{M}]_{\text {eq,aq }} /\left[[\mathrm{M}]_{\text {eq,aq }}\right.\right.
\end{aligned}
$$

To examine the relationship between $E \%$ and atomic number, an aqueous mixed solution containing La(III), Ce(III), Pr(III), Nd(III), Sm(III), Eu(III), Gd(III), Tb(III), Dy(III), Ho(III), Er(III), Tm(III), Yb(III), $\mathrm{Lu}(\mathrm{III})$ and $\mathrm{Y}(\mathrm{III})\left(1 \times 10^{-4} \mathrm{M}\right.$ each) was prepared as described above and adjusted to a certain $\mathrm{pH}$ value using $\mathrm{HCl} / \mathrm{ammonia}$ solutions. The metal concentrations were determined by ICP-MS (Perkin Elmer, NexION 300).

All extraction experiments were performed at $23 \pm 2{ }^{\circ} \mathrm{C}$. The volume ratio of the organic phase to 
the aqueous phase was 1 at all times.

\subsection{FT-IR analysis}

A sample for the Dy(III) complex extracted with CPCA was obtained as follows. First, Dy(III) was extracted with CPCA (organic phase: $0.1 \mathrm{M}$ CPCA in chloroform, aqueous phase: $0.05 \mathrm{M} \mathrm{Dy(III)} \mathrm{in} \mathrm{dilute}$ $\mathrm{HCl}$ solution). Then the organic phase was contacted three times with the fresh aqueous phase to afford an organic solution containing $0.02 \mathrm{M} \mathrm{Dy}(\mathrm{III}): \mathrm{pH}$ of the equilibrated aqueous phase was $1.8 \pm 0.1$. The Dy(III) extracted organic solution and the initial organic phase were deposited onto a ST-IR Card TYPE 1 (Thermo Electron Co.). A pellet of CPCA was also prepared for FT-IR analysis by grinding with $\mathrm{KBr}$. FT-IR spectra were collected using a PerkinElmer Spectrum 100 instrument.

\section{Results and Discussion}

\subsection{Effect of $\mathrm{pH}$ and CPCA concentration}

Figure $2 \mathrm{~A}$ shows the $D$ of $\mathrm{Nd}(\mathrm{III}), \mathrm{Eu}(\mathrm{III}), \mathrm{Dy}(\mathrm{III}), \mathrm{Lu}(\mathrm{III})$ and $\mathrm{Y}(\mathrm{III})$ as a function of $\mathrm{pH}$ of the equilibrated aqueous phase. The $\log D$ values of all the ions increased with increasing $\mathrm{pH}$ with slope of the plot remaining around 3 for all the ions, indicating that each $\mathrm{RE}(\mathrm{III})$ ion was neutralized by three deprotonated CPCA molecules; that is, the cation-exchange reaction occurred in this system.

Plots of $\log D-3 \mathrm{pH}$ versus $\log [\mathrm{CPCA}(\mathrm{M})]$ are shown in Figure 2B. The slope values remained around 3 for all the ions; thus, the extraction reaction can be described using the following equation.

$$
\mathrm{RE}(\mathrm{III})_{(\mathrm{aq})}+3 \mathrm{HL}_{(\mathrm{org})} \rightleftharpoons \mathrm{RE} \cdot \mathrm{L}_{3(\mathrm{org})}+3 \mathrm{H}^{+}{ }_{(\mathrm{aq})}
$$

(HL: CPCA, org: organic phase, aq: aqueous phase)

(A)

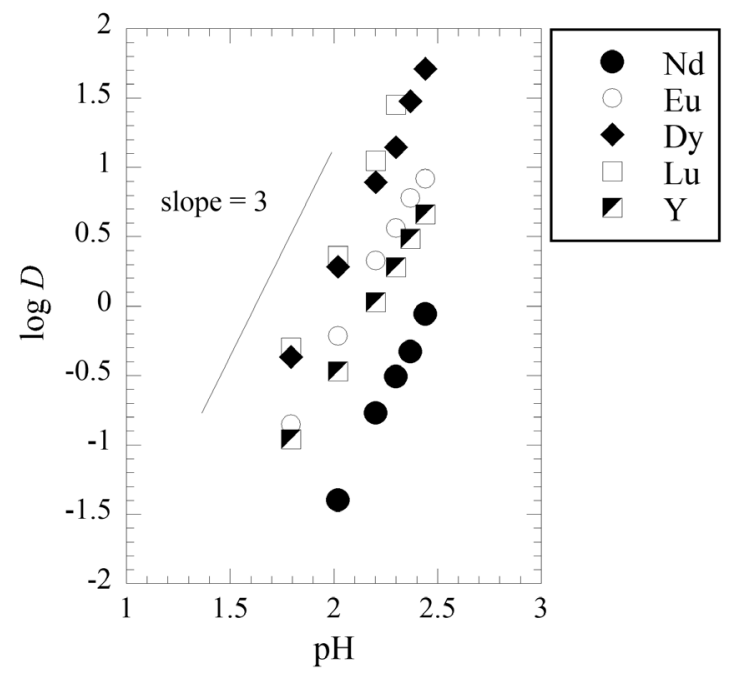

(B)

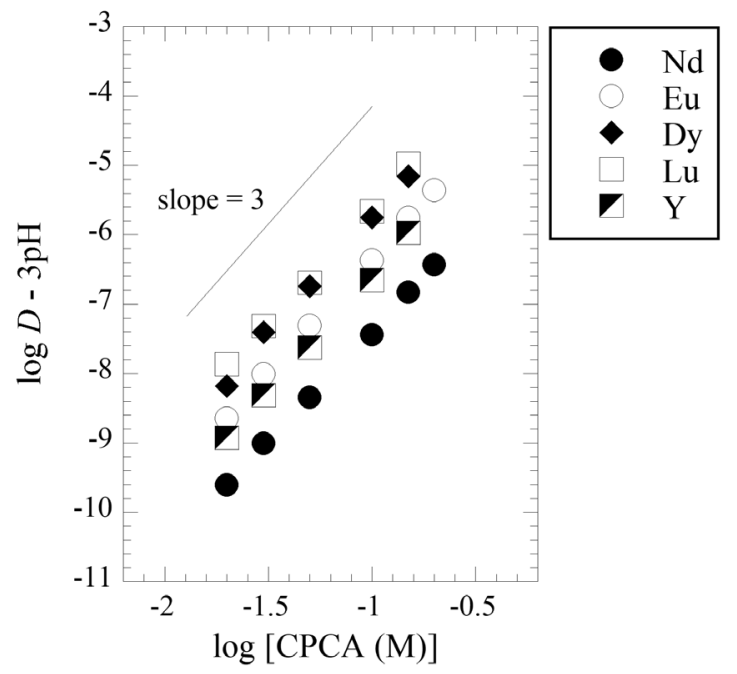

Figure 2. Plots of (A) $\log D$ versus $\mathrm{pH}$ of the equilibrated aqueous phase, and (B) $\log D-3 \mathrm{pH}$ versus $\log [\mathrm{CPCA}(\mathrm{M})]$. Organic phase for (A): $0.1 \mathrm{M}$ CPCA. Aqueous phase: $1 \mathrm{M}(\mathrm{H}, \mathrm{Na}) \mathrm{Cl}$ containing $\mathrm{Nd}(\mathrm{III}), \mathrm{Eu}(\mathrm{III}),\left(\mathrm{Dy}(\mathrm{III}), \mathrm{Lu}(\mathrm{III})\right.$, and Y(III) $\left(5 \times 10^{-4} \mathrm{M}\right.$ each). 
CPCA could not dimerize in the RE(III) complex, unlike acidic organophosphorus extractants, which form a dimer and extract RE(III) with 6 molecules (i.e., 3 dimers) [7]. The extraction reaction is the same as that seen in the DGAA system [13].

\subsection{Coordination mode of CPCA}

In order to understand the coordination property of CPCA to RE(III), FT-IR measurements were performed for the CPCA and Dy(III)-CPCA samples. We focused on the carbonyl stretching frequencies assigned to the carboxyl and amide groups. Figure 3 shows the FT-IR spectra in the range of 1500-1800 $\mathrm{cm}^{-1}$ for (a) CPCA in $\mathrm{KBr}$, (b) CPCA in chloroform and (c) Dy(III) complex extracted with CPCA in chloroform.

In the spectrum a, two intense peaks appeared at $1738 \mathrm{~cm}^{-1}$ and $1608 \mathrm{~cm}^{-1}$, which were assigned to the carbonyl stretching band $\left(v_{(\mathrm{CO})}\right)$ of the carboxyl and amide groups, respectively $[15,16]$; a peak at $1571 \mathrm{~cm}^{-1}$ was also observed, which could be from the aromatic ring vibrations $[17,18]$. In the spectrum $\mathbf{b}$, a new intense peak $\left(1733 \mathrm{~cm}^{-1}\right)$ and a shoulder peak (around $1630 \mathrm{~cm}^{-1}$ ) occurred. The former peak likely corresponds to the $v_{(\mathrm{CO})}$ of the carboxyl group that interacted with chloroform via hydrogen bonds [19]. The latter peak can be assigned to the asymmetric stretching vibration of the carboxylate $\left(v_{\text {as( }\left(\mathrm{COO}^{-}\right)}\right)[18,20]$, indicating that CPCA was partially deprotonated in chloroform, whereas the corresponding symmetric stretching vibration could not be identified in this spectrum.

Given that the 1:3 Dy(III)-CPCA complex was formed, the relative concentrations of the solutions used (0.1 M CPCA containing $0.02 \mathrm{M}$ Dy(III)) meant that about half of the CPCA molecules remained non-coordinated. Thus, in the spectrum c, the peaks appearing at nearly the

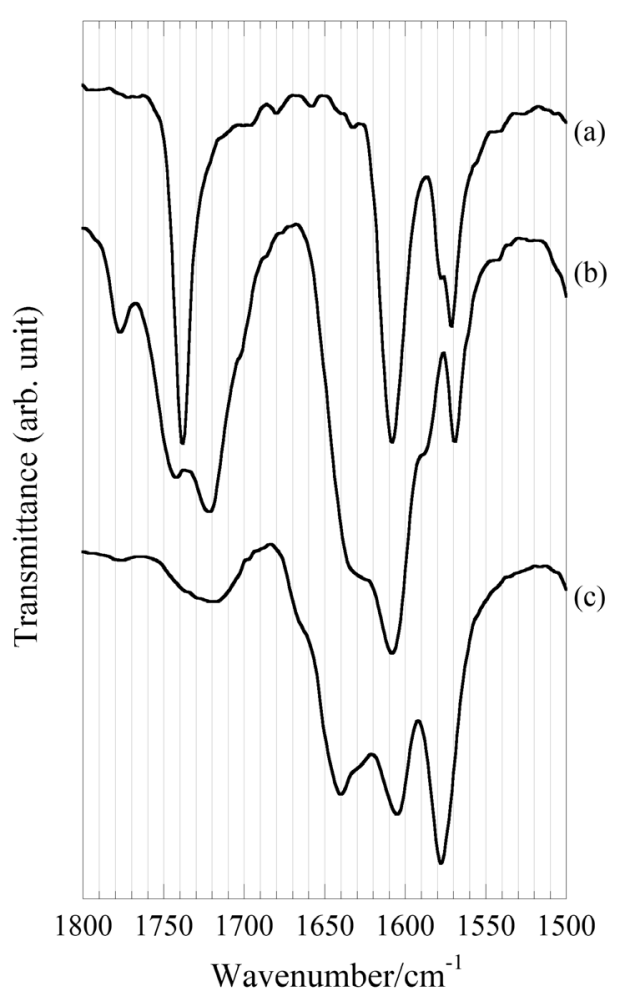

Figure 3. FT-IR spectra in the range of $1500-1800$ $\mathrm{cm}^{-1}$ for (a) $\mathrm{CPCA}$ in $\mathrm{KBr}$, (b) $\mathrm{CPCA}$ in chloroform and (c) Dy(III) complex extracted with CPCA in chloroform. same wavenumber as those in the spectrum b were considered to stem from the non-coordinated CPCA molecules. The intense peak at $1578 \mathrm{~cm}^{-1}$ probably corresponds to the $v_{(\mathrm{CO})}$ of the amide group, which shifted by approximately $30 \mathrm{~cm}^{-1}$ from that for the non-coordinated CPCA. This lower wavenumber shift has been observed in picolinamide complexes in which an oxygen atom of the amide group directly coordinates to a RE(III) ion [21]. In addition, a new peak appeared at $1640 \mathrm{~cm}^{-1}$. It has been reported from an analysis of RE(III) complexes with picolinic acid that coordination of the carboxylate to $\mathrm{RE}$ (III) results in a slight shift of the $v_{\mathrm{as}\left(\mathrm{COO}^{-}\right)}$peak to a higher wavenumber [18]. Accordingly, the obtained results clearly show coordination of the carboxyl and amide 
oxygen atoms to Dy(III). Although coordination of the pyridine nitrogen atom could not be clarified from the spectra, considering the structure of CPCA and the formation of a 1:3 Dy(III)-CPCA complex, we conclude that CPCA extracts Dy(III) via a tridentate mode.

\subsection{Lanthanide pattern and position of Y(III)}

Figure 4 shows the relationship between $E \%$ and lanthanide atomic number, termed the lanthanide pattern, as well as the $E \%$ of Y(III). $E \%$ rapidly increased with increasing atomic number until $\mathrm{Er}(\mathrm{III})$, with the exception of $\mathrm{Gd}(\mathrm{III})$, for which the gadolinium break was observed [22], and it then remained almost constant from $\operatorname{Er}(\mathrm{III})$ to $\mathrm{Lu}(\mathrm{III})$. This pattern is similar to that reported for a DGAA system [12]. The shape of the lanthanide pattern can be considered to reflect the characteristics of a RE(III) extraction system. It is well known that the decrease in ionic radii due to lanthanide contraction provides the increasing hard property with increasing atomic number; the increasing hard property corresponds to the increasing dehydration energy from $\mathrm{La}(\mathrm{IIII})$ to $\mathrm{Lu}(\mathrm{III})$ [23]. In addition, the coordination geometry of the extractants, (i.e., size effect) has a huge impact on the lanthanide pattern [24]. Thus, a hard donor-type extractant does not always show an $E \%$ that increases with atomic number $[10,25]$. In this regard, the similar lanthanide pattern between the CPCA and DGAA systems is consistent with these extractants having the similar coordination mode (i.e., tridentate) [26].

The position of $\mathrm{Y}(\mathrm{III})$ in the lanthanide series for extraction efficiency is highly dependent on the extraction systems [25]; For example, for acidic organophosphorus extractants, Y(III) is usually close to $\mathrm{Ho}(\mathrm{III})$, whereas, for carboxylic acid-type extractants, the position of Y(III) can vary in the middle of the lanthanide series for Versatic 10, in the light lanthanides for naphthenic acid, and beyond all the lanthanides for sec-nonylphenoxy acetic acid. In the CPCA system, Y(III) was close to Sm(III) as seen in Figure 4. This position is similar to that reported for a DGAA-Y(III) system (close to Eu(III)) [14]. This similarity also corresponds to almost the same coordination property between CPCA and DGAA.

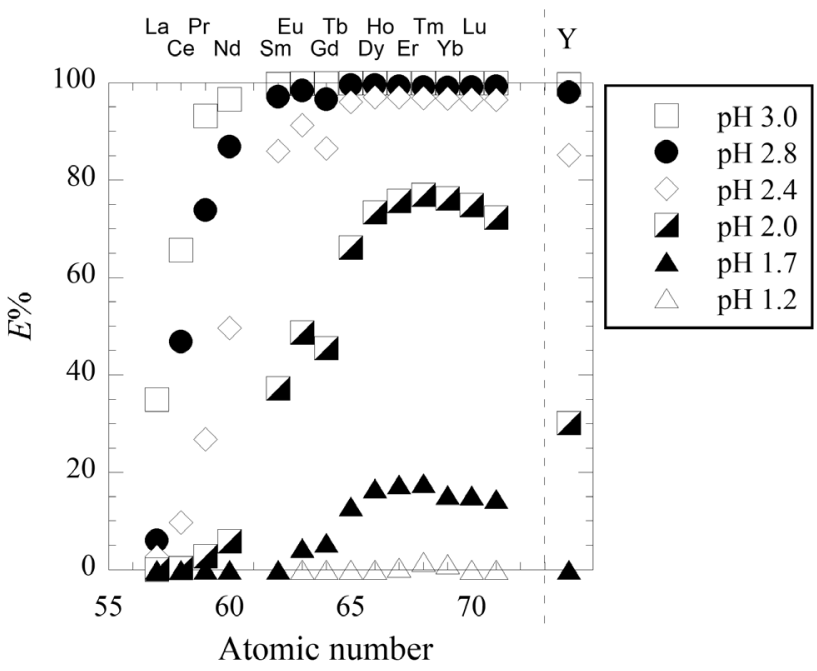

Figure 4. Relationship between $E \%$ and atomic number. Organic phase: 0.1 M CPCA. Aqueous phase: dilute $\mathrm{HCl}$ solution containing $\mathrm{La}(\mathrm{III}), \mathrm{Ce}(\mathrm{III}), \operatorname{Pr}(\mathrm{III}), \mathrm{Nd}(\mathrm{III})$, $\mathrm{Sm}(\mathrm{III}), \mathrm{Eu}(\mathrm{III}), \mathrm{Gd}(\mathrm{III}), \mathrm{Tb}(\mathrm{III}), \mathrm{Dy}(\mathrm{III}), \mathrm{Ho}(\mathrm{III}), \mathrm{Er}(\mathrm{III})$, $\mathrm{Tm}(\mathrm{III}), \mathrm{Yb}(\mathrm{III}), \mathrm{Lu}(\mathrm{III})$, and $\mathrm{Y}(\mathrm{III})\left(1 \times 10^{-4} \mathrm{M}\right.$ each $)$.

From Figure 4, the separation factor of $D_{\mathrm{Nd}} / D_{\mathrm{Pr}}$ at $\mathrm{pH} 2.0-3.0$ was $2.3 \pm 0.3$. This value is similar to that reported for a DGAA system [26], but larger than that in the commercial organophosphorus systems using D2EHPA and HEHEHP [1]. 


\section{Conclusion}

The extraction properties of RE(III) were investigated with a new acidic amide compound, CPCA. Slope analysis of $D$ revealed that three deprotonated CPCA molecules extracted a RE(III) ion for all the RE(III) studied. Peak shifts in the FT-IR spectra suggested that CPCA molecules coordinated to Dy(III) in a tridentate mode. The lanthanide pattern showed an increase in extraction efficiency from $\mathrm{La}(\mathrm{III})$ to $\mathrm{Er}(\mathrm{III})$. These findings are similar to those reported for DGAA systems, indicating that CPCA and DGAA have similar coordination properties to $\mathrm{RE}(\mathrm{III})$.

\section{Acknowledgement}

The authors wish to thank Dr. Shinichi Suzuki (Japan Atomic Energy Agency) for the ICP-MS measurements, and Ms. Tomoko Saito and Ms. Hiroko Niiyama (AIST) for technical assistance. A part of this study was financially supported by the NEDO project "Research and Development of Recycling Technologies for Establishing a High Efficiency Resource Circulation System".

\section{References}

1) C. K. Gupta, N. Krishnamurthy, "Extractive Metallurgy of Rare Earth", CRC Press. (2006).

2) K. Binnemans, P. T. Jones, B. Blanpain, T. V. Gerven, Y. Yang, A. Walton, M. Buchert, J. Cleaner Prod., 51, 1-22 (2013).

3) M. Tanaka, T. Oki, K. Koyama, H. Narita, T. Oishi, in "Handbook on the Physics and Chemistry of Rare Earths, Vol. 43", Elsevier B. V., Amsterdam, pp. 159-211 (2013).

4) Y. Ding, D. Harvey, N.-H. L. Wang, Green Chem., 22, 3769-3783 (2020).

5) L. Sherrington, in "Handbook of Solvent Extraction", John Wiley \& Sons, Inc., New York, Chapter 25.6, pp. 717-723 (1983).

6) M. Cox, in "Solvent Extraction Principles and Practices", Marcel Dekker, Inc., New York, Chapter 11, pp. 455-505 (2004).

7) F. Xie, T. A. Zhang, D. Dreisinger, F. Doyle, Miner. Eng., 56, 10-28 (2014).

8) H. Narita, T. Yaita, K. Tamura, S. Tachimori, Radiochim. Acta, 81, 223-226 (1998).

9) H. Narita, T. Yaita, K. Tamura, S. Tachimori, J. Radioanal. Nucl. Chem., 81, 381-384 (1999).

10) H. Narita, T. Yaita, S. Tachimori, Solvent Extr. Ion Exch., 22, 135-145 (2004).

11) A. Shimada, T. Yaita, H. Narita, S. Tachimori, K. Okuno, Solvent Extr. Ion Exch., 22, 147-161 (2004).

12) H. Naganawa, K. Shimojo, H. Mitamura, Y. Sugo, J. Noro, M. Goto, Solvent Extr. Res. Dev., Jpn., 14, 151-159 (2007).

13) K. Shimojo, N. Aoyagi, T. Saito, H. Okamura, F. Kubota, M. Goto, H. Naganawa, Anal. Sci., 30, 263-269 (2014).

14) K. Shimojo, A. Nakai, H. Okamura, T. Saito, A. Ohashi, H. Naganawa, Anal. Sci., 30, 513-517 (2014).

15) A. Rout, K. Binnemans, Ind. Eng. Chem. Res., 53, 6500-6508 (2014).

16) A. S. Suneesh, K. A. Venkatesan, K. V. Syamala, M. P. Antony, P. R. Vasudeva Rao, Radiochim. Acta, 100, 425-430 (2012).

17) F.-L. Hu, X.-H. Yin, Y. Mi, S.-S. Zhang, W.-Q. Luo, Spectrochim. Acta, Part A, 75, 825-829 (2010).

18) G. Świderski, M. Kalinowska, J. Malejko, W. Lewandowski, Vib. Spectrosc., 87, 81-87 (2016). 
19) R. A. Nyquist, T. D. Clark, R. Streck, Vib. Spectrosc., 7, 275-286 (1994).

20) J. Kumanotani, Yukagaku, 11, 577-590 (1962).

21) J. Xue, Y. Jiang, W. Li, L. Yang, Y. Xu, G. Zhao, G. Zhang, X. Bu, K. Liu, J. Chen, J. Wu, Spectrochim. Acta, Part A, 137, 864-870 (2015).

22) H. Yamada, M. Miyashita, Y. Sekiguchi, M. Kojima, H. Wada, Anal. Sci., 14, 703-711 (1998).

23) K. L. Nash, Solvent Extr. Ion Exch., 11, $729-768$ (1993).

24) M. R. Healy, A. S. Ivanov, Y. Karslyan, V. S. Bryantsev, B. A. Moyer, S. Jansone-Popova, Chem. Eur. J., 25, 6326-6331 (2019).

25) D. Li, J. Rare Earths, 35, 107-119 (2017).

26) S. M. Ibrahim, Y. Zhang, Y. Xue, S. Yang, F. Ma, Y. Gao, Y. Zhou, G. Tian, ACS Omega, 4, 20797-20806 (2019). 